\title{
Thymine Dimerization Induced by Oxidative DNA Lesions and Epigenetic Intermediates via Triplet-Triplet Energy Transfer
}

\author{
Mauricio Lineros-Rosa, a Antonio Francés-Monerris, ${ }^{\mathrm{b}, \mathrm{c}}$ Antonio Monari, ${ }^{*, \mathrm{~b}}$ Miguel Angel Miranda, ${ }^{*}$ a \\ and Virginie Lhiaubet-Vallet*,a
}

aInstituto Universitario Mixto de Tecnologia Química UPV-CSIC, Universitat Politècnica de València, Avda de los Naranjos s/n, 46022 Valencia, Spain.

bUniversité de Lorraine and CNRS, LPCT UMR 7019, F-54000 Nancy, France.

‘Departament de Química Física, Universitat de València, 46100 Burjassot, Spain

KEYWORDS. UV light, DNA damage, cyclobutane pyrimidine dimer, time-resolved spectroscopy, CASPT2, density functional theory, exciplex, DNA oxidation, formyluracil, formylcytosine

\begin{abstract}
Interaction of nucleic acids with light is a scientific question of paramount relevance not only in the understanding of life functioning and evolution, but also in the insurgence of diseases such as malignant skin cancer and in the development of biomarkers and novel light-assisted therapeutic tools. This work shows that the UVA portion of sunlight, not absorbed by canonical DNA nucleobases, can be absorbed by 5 -formyluracil (ForU) and 5-formylcytosine (ForC), two ubiquitous oxidative lesions and epigenetic intermediates present in living beings in natural conditions. We measure the strong propensity of these molecules to populate triplet excited states able to transfer the excitation energy to thymine-thymine dyads, inducing the formation of the highly toxic and mutagenic cyclobutane pyrimidine dimers (CPDs). By using steady-state and transient absorption spectroscopy, NMR, HPLC, and theoretical calculations, we quantify the differences in the triplet-triplet energy transfer mediated by ForU and ForC, revealing that the former is much more efficient in delivering the excitation energy and producing the CPD photoproduct. Although significantly slower than ForU, ForC is also able to harm DNA nucleobases and therefore this process has to be taken into account as a viable photosensitization mechanism. The present findings evidence a rich photochemistry crucial to understand DNA photodamage and of potential use in the development of biomarkers and nonconventional photodynamic therapy agents.
\end{abstract}

\section{INTRODUCTION}

Nucleic acids are constantly exposed to endogenous and exogenous agents that can modify their chemical structure and therefore compromise their normal biological function..$^{1,2}$ Among such agents, solar light and especially UVB radiation ( 290-320 $\mathrm{nm})$ at high doses represents a major threat to public health. As a matter of fact, ca. $5-10 \%$ of the total UV radiation that reaches Earth's surface falls in this wavelength range ${ }^{3}$ and DNA nucleobases directly absorb UVB wavelengths, triggering complex photochemical pathways leading to the appearance of DNA lesions. ${ }^{4-11}$ Although DNA nucleobases are known for their high photostability, ${ }^{4-}$ 8 dangerous DNA modifications such as cyclobutane pyrimidine dimers (CPDs) and 6-4 photoproducts (64-PP) are constantly produced and may accumulate in the skin, inducing mutation and carginogenesis. ${ }^{12,13}$ The unprotected exposure to UV light is nowadays recognized as one of the main causes of malignant skin cancer such as melanoma. ${ }^{14-16}$

The toxicity of DNA photolesions has been extensively studied: whereas CPDs are the most abundant photoproducts and are highly resistant to repair, 64-PP are less common, better repaired, but extremely mutagenic. ${ }^{17}$ The high repair resistance of CPDs also results in their accumulation at the genome level. Furthermore, the knocking down of photolesions nucleotide excision repair (NER) enzymes is the main cause of highly debilitating pathologies, such as Xeroderma Pigmentosum, and of the exponential increase of skin cancer predisposition.

The rest of UV solar radiation, which is not filtered by the ozone layer $(\sim 90 \%)$, belongs to the less energetic UVA wavelengths $(\sim 320-380 \mathrm{~nm}) .^{3}$ In contrast to UVB photons, UVA light is hardly absorbed by DNA nucleobases. Instead, it can be absorbed by different chromophores, either exogenous or endogenous, located spatially close to DNA, which in turn can produce DNA lesions by indirect photochemical processes in what is known as DNA photosensitization. ${ }^{18}$ These usually involve the population of triplet states of the chromophores by means of intersystem crossing (ISC) followed by several photochemical routes traditionally categorized in three classes: $i$ ) triplet-triplet energy transfer (TTET) to DNA, ii) activation of molecular oxygen to its singlet excited state, and iii) photoinduced electron transfer processes usually involving guanine. Other mechanisms such as photodissociation or hydrogen abstraction have also been reported. ${ }^{4,19}$ While electron transfer and singlet oxygen activation usually produce oxidative DNA lesions, 
triplet-triplet energy transfer has been shown, both computationally and experimentally, to favor thymine dimerization. ${ }^{12,20,21}$ Here we focus on the first class of photosensitization, i.e. the triplet-triplet energy transfer from the chromophore to DNA.

A wide range of external photosensitizers have been studied in the last years, ${ }^{12,22-25}$ a representative example being the paradigmatic case of benzophenone. ${ }^{26-28}$ Nevertheless, it has been recently proposed that DNA lesions can themselves act as photosensitizers, since the modifications of their chemical structure may alter their optical and photophysical properties. This phenomenon was named as "Trojan Horse" after recognizing that 64-PP acts as an internal photosensitizer. ${ }^{29}$ It was found that the presence of the pyrimidone moiety leads to absorption in the UVA range and induces CPD production after ISC and subsequent triplettriplet energy transfer. The possibility of triplet transfer was also confirmed using molecular modeling and simulations. ${ }^{30}$

Recently, the role of oxidative lesions, namely 5-formyluracil (ForU) ${ }^{31}$ and 5-formylcytosine (ForC) ${ }^{32,33}$ (see Scheme 1), as potential Trojan Horses has been studied through experiments $^{34}$ and theoretical calculations. ${ }^{35-37}$ It has indeed been recognized, both in model systems and in DNA oligomers, that the inclusion of ForU is correlated with a significant increase in the yield of CPD damages. ${ }^{34}$ Furthermore, molecular modeling and simulations have shown that ForU is prone to a facile ISC, leading to an energetically favorable TTET to thymine and that those conditions are maintained in the B-DNA environment. ${ }^{35}$ Subsequently, it was shown that ISC is also possible in the case of ForC, even if non-adiabatic molecular dynamics have pointed out a less-favorable phenomenon as compared to ForU. ${ }^{37}$

The behavior of ForC in comparison with ForU is indeed extremely intriguing, not only from a photophysical perspective, but also due to its biological relevance. ForC can be considered an oxidative lesion, but also an epigenetic intermediate in the demethylation process of 5-methyl-cytosine (5MetC). Indeed, the methylation of cytosine at 5 position, occurring in non-coding DNA regions with a high density of guanine and cytosine, the so-called CpG islands, results in the silencing of the specific gene expression. In eukaryotic cells, the regulation of the gene expression as a response to external stress is controlled by DNA methyltransferases (DNMT) that provide an oxidative route to demethylation involving the oxidation of $5 \mathrm{MetC}$ to ForC, and the subsequent participation of the base excision repair (BER) machinery. Deregulation in gene expression is found in many diseases including cancers, therefore, epigenetics intermediates are also recognized as most valuable biomarkers. The fact that an epigenetic intermediate, ForC, can potentially induce DNA photolesions, clearly opens fundamental questions concerning the subtle interplay between epigenetic phenomena and DNA photolesions, and hence can contribute to solve the molecular grounds of the problems related to the cellular tumorigenesis and the protective mechanisms that cells may have developed as a result of evolutionary pressure.

In this article, we rationalize the propensity of ForC to photoinduce thymine dimerization in model systems and DNA oligomers, also comparing the process with the one operative in the case of ForU. The crucial differences between the two chromophores and their effects on thymine dimerization are also duly analyzed. Indeed, the description of these processes at a molecular and electronic level is of utmost importance to comprehend the naturally occurring mechanisms of DNA damage. Moreover, it also paves the way in the development of therapeutic tools such as photodynamic therapy, in which the DNA of cancerous cells in presence of man-made photosensitizers is selectively harmed by using visible or IR light.

\section{RESULTS AND DISCUSSION}

\section{Triplet excited states of ForU and ForC}

The photochemistry of ForU and ForC (Scheme 1) was tackled in aqueous media at room temperature in order to evaluate their potential to act as intrinsic photosensitizers. In this context, triplet excited states have been well-established as key intermediates in the photosensitized generation of damages. ${ }^{12,38}$ Therefore, in addition to the spectroscopic characterization of the formyl derivatives, particular attention was paid here to thymine dimer formation as an analytical tool to compare the harmfulness of ForU and ForC triplet excited states. Indeed, the photosensitization of cyclobutane thymine dimer (Thy<>Thy, Scheme 1) is a clean reaction that occurs by TTET from the photosensitizer excited state to the pyrimidine base. To be efficient, this process requires a photosensitizer with a high triplet energy (higher than that of thymine), a high ISC quantum yield to allow significant population of the triplet manifold, and a triplet lifetime long enough to maximize the likelihood of the energy transfer process. Hence, monitoring Thy<> Thy formation provides valuable information on the overall strength of photosensitizing compounds.

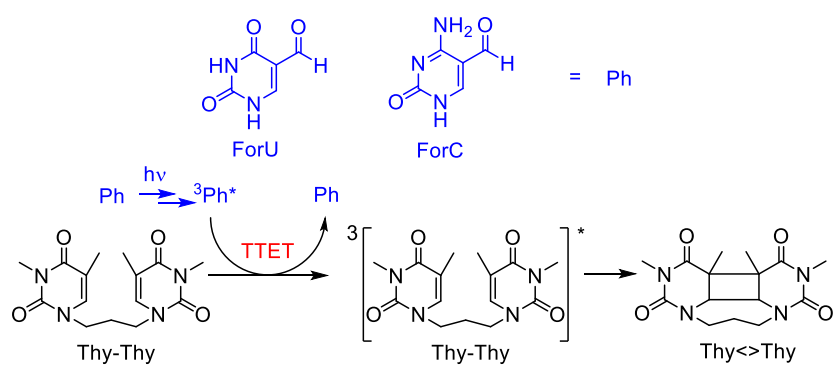

Scheme 1. Structures of both photosensitizers $(\mathrm{Ph})$ under study (ForU and ForC) and the photosensitized [2+2] photocycloaddition of Thy-Thy to afford Thy<> Thy

The first important property of an efficient DNA photosensitizer is absorption of light in a wavelength region where nucleobases do not (or barely) absorb, to assure the exploitation of the maximum number of incoming photons. As shown in Figure $\mathrm{S} 3$, in $\mathrm{MeCN}: \mathrm{H}_{2} \mathrm{O}(1: 1, \mathrm{v}: \mathrm{v})$, ForC and ForU share similar absorption maxima $\left(\lambda_{\max }\right)$ at $276-280 \mathrm{~nm}$. Both compounds exhibit a band that reaches the UVA region allowing their selective excitation in the presence of canonical DNA bases. Nonetheless, the uracil derivative has a larger band, which broadens the spectral range available for 
its excitation. None of the compounds show fluorescence emission, pointing toward an efficient deactivation of the singlet excited state by nonradiative pathways such as internal conversion or ISC. By contrast, phosphorescence was detected in ethanol glass at $77 \mathrm{~K}$ with a maximum at 445 and $425 \mathrm{~nm}$ for ForU and ForC, respectively (Figure S4). This emission informs not only on the occurrence of ISC, which populates the triplet excited state, but also on the triplet energy $\left(\mathrm{E}_{\mathrm{T}}\right)$ of the uracil and cytosine derivatives determined at ca. 3.67 and $3.94 \mathrm{eV}$, respectively. ${ }^{37}$ The triplet excited state behavior at room temperature was monitored using laser flash photolysis, since this technique allows the detection and characterization of transient species absorbing between 300 and $700 \mathrm{~nm}$ and whose lifetimes are typically in the microsecond timescale. Thus, nitrogen bubbled solutions in $\mathrm{H}_{2} \mathrm{O}$ :MeCN (1:1) of the two formyl derivatives were excited at $266 \mathrm{~nm}$ using the $4^{\text {th }}$ harmonic of a Nd:YAG laser. The transient spectrum of ForU (Figure 1) showed a single broad band with maximum at 440-460 nm that completely disappeared after $40 \mu$ s without giving rise to any other species. This transient signal was assigned to the triplet-triplet absorption by comparison with our previously published data in PBS, but the spectrum in aqueous acetonitrile was cleaner and much less noisy. ${ }^{34}$ The decay monitored at 440 $\mathrm{nm}$ can be fitted with a monoexponential function, $\mathrm{f}(\mathrm{t})=\mathrm{A}$ $\exp (-t / \tau)$, yielding a lifetime $\tau$ of ca. $12 \mu$ s (Figure 1, inset) that is much longer than the one found in PBS.

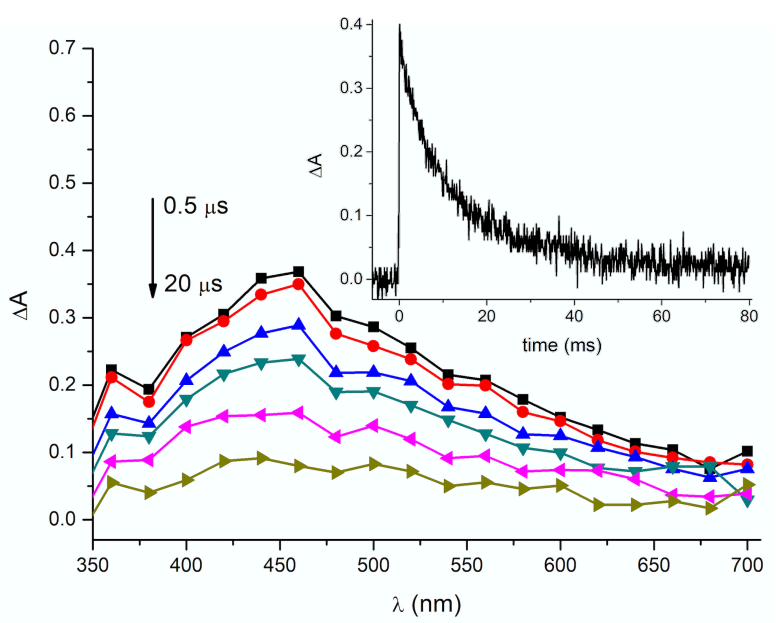

Figure 1. Transient absorption spectra of 5-formyluracil in MeCN: $\mathrm{H}_{2} \mathrm{O}(1: 1, \mathrm{v}: \mathrm{v})$ under $\mathrm{N}_{2}$ at different times after the $266 \mathrm{~nm}$ laser pulse. Inset: decay of ForU monitored at 440 $\mathrm{nm}$.

The cytosine derivative, ForC, also displayed a signal centered at $440 \mathrm{~nm}$ together with a shoulder at $560 \mathrm{~nm}$, both bands decreasing with the same kinetics (Figure 2); this transient signal was assigned to the triplet excited state of ForC. ${ }^{36}$ Concerning the kinetics, a biexponential behavior was found for the 440 and $560 \mathrm{~nm}$ decays, which were adjusted using the equation $f(t)=A_{1} \exp \left(-t / \tau_{1}\right)+A_{2} \exp \left(-t / \tau_{2}\right)$. The obtained lifetimes are $\tau_{1}=0.07 \mu \mathrm{s}\left(\mathrm{A}_{1}=0.25\right)$ and $\tau_{2}=2.3$ $\mu \mathrm{s}\left(\mathrm{A}_{2}=0.75\right)$ (Figure 2, inset). Both characteristic lifetimes are significantly shorter than that of ${ }^{3}$ ForU*, and thus, the dynamic range for ${ }^{3} \mathrm{ForC}^{*}$ intermolecular quenching is reduced, especially in the case of $\tau_{1}$. Thus, bimolecular rate constants for ${ }^{3} \mathrm{ForC}^{*}$ quenching were further determined using the changes of $\tau_{2}$, only.

These intrinsic differences in the triplet lifetimes correlate very well with the nature of the emissive triplet states predicted by previous calculations for the two monomers. The emissive triplet states have been identified as ${ }^{3} n \pi^{*}$ and ${ }^{3} \pi \pi^{*}$, for ForU and ForC respectively, on the basis of their vertical emissions.$^{37}$ Thus, the ${ }^{3} n \pi^{*}$ state of ForU has a longer lifetime given the lowest transition dipole moment with the singlet ground state and hence a smaller oscillator strength, resulting, following Einstein thumb rule, in a slower radiative decay as compared to the ${ }^{3} \pi \pi^{*}$ of ForC. ${ }^{37}$

The above results establish that, upon excitation, both ForU and ForC populate triplet excited states with energies higher than that of thymine and with a lifetime in the microsecond timescale, hence they can potentially induce energy transfer. Thus, the next step was to evaluate the ability of these excited states to photosensitize CPDs formation by means of time-resolved and steady-state experiments.

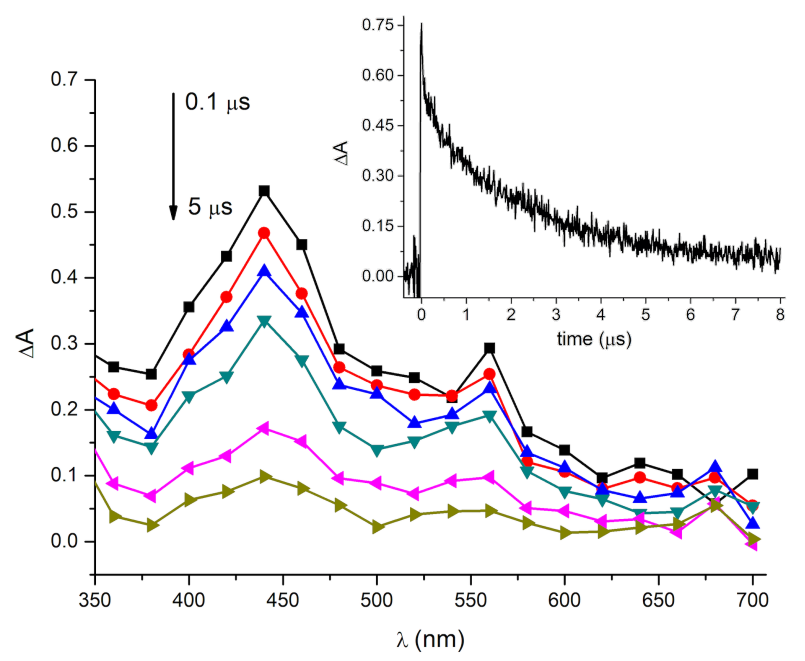

Figure 2. Transient absorption spectra of 5-formylcytosine in $\mathrm{MeCN}: \mathrm{H}_{2} \mathrm{O}(1: 1, \mathrm{v}: \mathrm{v})$ under $\mathrm{N}_{2}$ at different times after the $266 \mathrm{~nm}$ laser pulse. Inset: decay of ForC monitored at 440 nm.

\section{Triplet-triplet energy transfer rates to Thy-Thy measured by laser flash photolysis}

First, laser flash photolysis was employed to determine the rate of the triplet-triplet energy transfer from ${ }^{3}$ ForU* ${ }^{*}$ and ${ }^{3}$ ForC* to Thy-Thy (Scheme 1), used as a model of adjacent nucleobases. As shown in Figure 3 (and Figure S5), the addition of increasing amounts of Thy-Thy resulted in the shortening of the triplet excited state decay kinetics of the formyl derivatives, together with a decrease of the end-ofpulse signal intensity, due to the filter effect produced by the thymine chromophore at the excitation wavelength. Stern-Volmer plot representation of $\tau_{0} / \tau$ as a function of the quencher concentration, reported as inlay, provided the bimolecular TTET rate constant $\left(\mathrm{k}_{\mathrm{q}}\right)$ for both sensitizers (see Material and Methods for more details). A value of $1.3 \times 10^{9}$ 
$\mathrm{M}^{-1} \mathrm{~S}^{-1}$ was obtained for the ${ }^{3} \mathrm{For} \mathrm{U}^{*}$ quenching, while the process was almost 3 times slower for ${ }^{3} \mathrm{ForC}^{*}$ with a $\mathrm{k}_{\mathrm{q}}$ of $c a .4 .6$ $\mathrm{x} 10^{8} \mathrm{M}^{-1} \mathrm{~s}^{-1}$. Taking into account that the model system has two thymine units, the obtained values are in agreement with those previously given in the literature for TTET processes involving thymine as energy acceptor using other photosensitizers. ${ }^{12,39}$

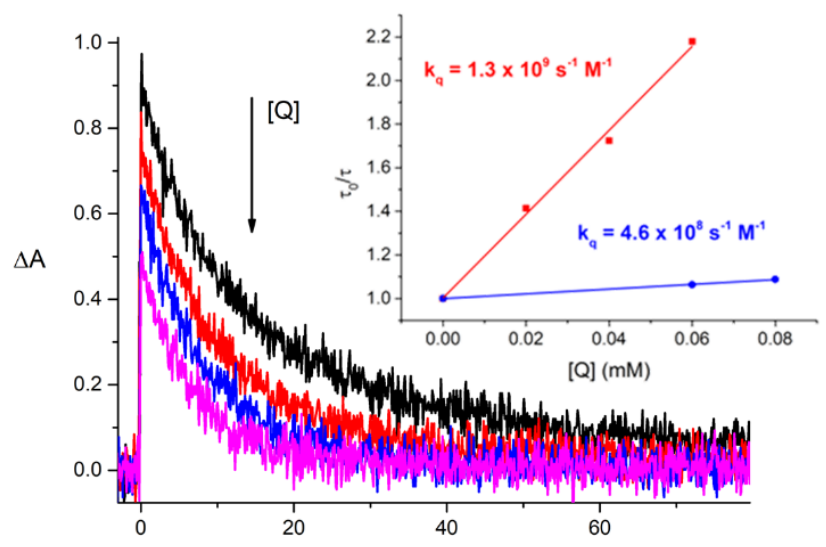

Figure 3. Decays of ForU in deaerated $\mathrm{MeCN}: \mathrm{H}_{2} \mathrm{O}(1: 1, \mathrm{v}: \mathrm{v})$ at $440 \mathrm{~nm}$ after the laser pulse at $266 \mathrm{~nm}$ in the presence of different Thy-Thy concentrations (from 0 to $0.08 \mathrm{mM}$ ). Inset: Stern-Volmer quenching of ForC (blue) and ForU (red) by Thy-Thy.

Curiously, an inverted order for the rate constants was expected based on the triplet energies, higher for ForC than for ForU, and on the Sandros' equation.40 This equation establishes that, for triplet-triplet energy transfer processes, the larger the energy difference between the donor (ie. formyl derivatives) and the acceptor (ie. the Thy-Thy dyad), the higher the rate constant. The data obtained here pointed toward the occurrence of a more complex process than an typical energy transfer.

Cyclobutane pyrimidine dimer production monitored by NMR and HPLC

Next, steady-state photolysis was performed on deaerated MeCN: $\mathrm{H}_{2} \mathrm{O}(1: 1, \mathrm{v}: \mathrm{v})$ solutions of Thy-Thy in the presence of ForU or ForC and using monochromatic light at $\lambda_{\text {exc }}=310$ $\mathrm{nm}$. All samples contained the same concentration of ThyThy (3.5 mM), ForC concentration was fixed at $1 \mathrm{mM}$, while in the case of ForU, the photosensitizer concentration was adjusted in order to obtain the same absorbance at $\lambda_{\text {exc }}$ as for ForC.

The course of the photoreaction was followed by ${ }^{1} \mathrm{H}$ NMR and HPLC (Figures 4 and S6-S8). In both cases, a clean process was observed with the formation of the cis-syn cyclobutane dimer of Thy-Thy (Thy<> Thy) as the only photoproduct. This assignment was based on the comparison with the HPLC elution time and with the NMR chemical shifts of synthetized Thy<> Thy (see Figure S6 and S8, up).

The NMR spectra of irradiated samples gave relevant information on the course of the photoreaction and on the nature of the photoproducts. They show that the [2+2] photocycloaddition leading to Thy<> Thy formation results in the saturation of the C5-C6 double bond and induces characteristic changes in the chemical shifts $(\delta)$ of the protons belonging to the nucleobase and to the trimethylene bridge. The most pronounced change was observed for the H6 proton that shifted from 7.90 to upper fields at ca. $4.48 \mathrm{ppm}$ (Figure S6). Signals of the methyl group at N3 also experienced shielding passing from 3.77 to $3.51 \mathrm{ppm}$, while those of the C5 methyl moved from 2.38 to $2.00 \mathrm{ppm}$. The ratio of the integrals of these signals (taken as pairs) can be used to evaluate the reaction course. In the case of ForC irradiation, it was observed that after $1 \mathrm{~h}, 50 \%$ of Thy-Thy was decomposed to yield Thy<> Thy as the sole product (Figure S7, up). Interestingly, when using ForU as a photosensitizer, the initial dyad is completely consumed under the same conditions (Figure S7, bottom).

The HPLC analysis provided similar results. Figure 4A shows the chromatograms registered for different irradiation times of ForC in presence of Thy-Thy. The peak corresponding to the Thy-Thy signal, that is eluting at $12 \mathrm{~min}$, decreases concomitantly with the appearance and the increase of a new peak corresponding to Thy <> Thy ( $8 \mathrm{~min}$ ). A qualitatively analogous behavior was observed for the irradiation of the mixture ForU:Thy-Thy (Figure S8, up). A control experiment performed with Thy-Thy alone irradiated at $\lambda_{\text {exc }}=310 \mathrm{~nm}$ (Figure S8, bottom) was also carried out and confirmed the lower yields for the direct CPD formation in the absence of photosensitizer. As expected from the difference in the bimolecular rate constants, Thy-Thy was more efficiently consumed when ForU is acting as photosensitizer. This is clearly illustrated in Figure 4B showing that the complete consumption of Thy-Thy was achieved after $1 \mathrm{~h}$ of irradiation in presence of ForU, while only $50 \%$ of Thy-Thy is depleted in the presence of ForC, and $20 \%$ without any sensitizer.

Altogether, these experimental results showed that in spite of its higher triplet excited state energy, ForC is a less efficient Thy<> Thy photosensitizer than ForU. Thus, in order to shed more light on the molecular aspects responsible for this difference, molecular modelling was performed for a mixture of each formyl derivative and thymine.

\section{Mechanistic aspects revealed by molecular modelling}

Several factors explain the different yields of triplet-triplet energy transfer and CPD formation induced by ForU and ForC. First, the population of the triplet excited state is slower for ForC than for ForU, as shown by the respective time constants, $\tau_{\mathrm{ISC}}=1940.5$ and $3559.4 \mathrm{fs}$, respectively) determined by non-adiabatic molecular dynamics. ${ }^{37}$ Second, the triplet state of ForC decays faster, as evidenced by the time-resolved spectroscopic data shown in Figures 1 and 2, in coherence with the nature of the lowest triplet state $\left({ }^{3} n \pi^{*}\right.$ and ${ }^{3} \pi \pi^{*}$ for ForU and ForC, respectively). ${ }^{37}$ Finally, the potential energy landscape of the electronic states involved in the photoprocess are different for ForU-Thy and ForC-Thy systems, as shown in Figure 5. 


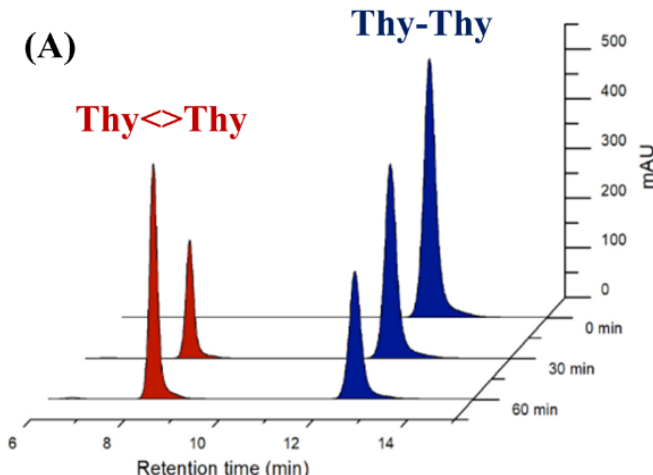

(B)

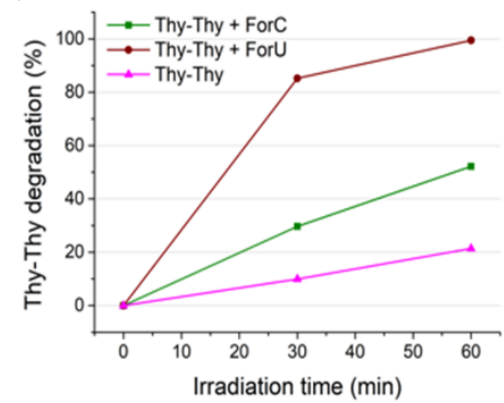

Figure 4. (A) HPLC chromatograms registered at $240 \mathrm{~nm}$ of ForC:Thy-Thy (1 mM: $3.5 \mathrm{mM})$ in $\mathrm{H}_{2} \mathrm{O}: \mathrm{MeCN}(1: 1, \mathrm{v}: \mathrm{v})$ irradiated from 0 to $60 \mathrm{~min}$ at $310 \mathrm{~nm}$, (B) Time-dependent photodegradation of Thy-Thy, in the presence of ForU (red), ForC (green) and alone (pink) $\left(\lambda_{\text {irr }}=310 \mathrm{~nm}\right)$.

Note that Figure 5 provides an overview of the energetic level of the most important electronic states calculated for relevant points of the potential energy surfaces, namely Franck-Condon, i.e. the ground state equilibrium geometry, and the optimized structures of the lowest singlet and triplet states.

In case of ForC, (Figure 5A) and as in the case of the monomer, ${ }^{37}$ the first excited singlet state, of ${ }^{1} \mathrm{n}, \pi^{*}$ nature and localized over ForC $\left({ }^{1} \mathrm{n}, \pi^{*}\right.$ Forc $)$, lies, at its equilibrium geometry, $0.48 \mathrm{eV}$ below the triplet state of ${ }^{3} \pi, \pi^{*}$ Forc nature, i.e. the other player involved in the ISC, justifying the slower population of the triplet manifold. Notably, and again coherently with non-adiabatic dynamics performed for the monomer, ${ }^{37}$ spin-orbit couplings corroborate the role of ${ }^{3} \pi, \pi^{*}$ ForC state as the doorway to ISC (Table S1). In contrast, in ForU, the corresponding states $\left({ }^{1} \mathrm{n}, \pi^{*}\right.$ ForU and ${ }^{3} \pi, \pi^{*}$ ForU $)$ are quasi-degenerated at the ${ }^{1} \mathrm{n}, \pi^{*}$ Foru equilibrium geometry, leading to a much more efficient triplet population (Figure 5B). ${ }^{35}$

Other significant differences between the two sensitizers are due to the distribution of the spin density between the couple photosensitizer-nucleobase. The triplet states of ForC-Thy are characterized by a marked delocalization of the $\pi$ clouds, describing the unpaired electrons, over both ForC and thymine moieties, especially involving the antibonding $\pi^{*}$ orbitals (see Figure 6). Hence, the triplet states can be classified as exciplex $\left(\mathrm{EXC}_{1}, \mathrm{EXC}_{2}\right)$. The delocalization is especially pronounced at the $\mathrm{S}_{0} \min$ and the $\mathrm{T}_{1} \mathrm{~min}$, that in diabatic notation is labelled as ${ }^{3} \mathrm{EXC}$ min. On the contrary, in the case of ForU, the spin density is much better localized over the two moieties, also giving rise to two well distinct

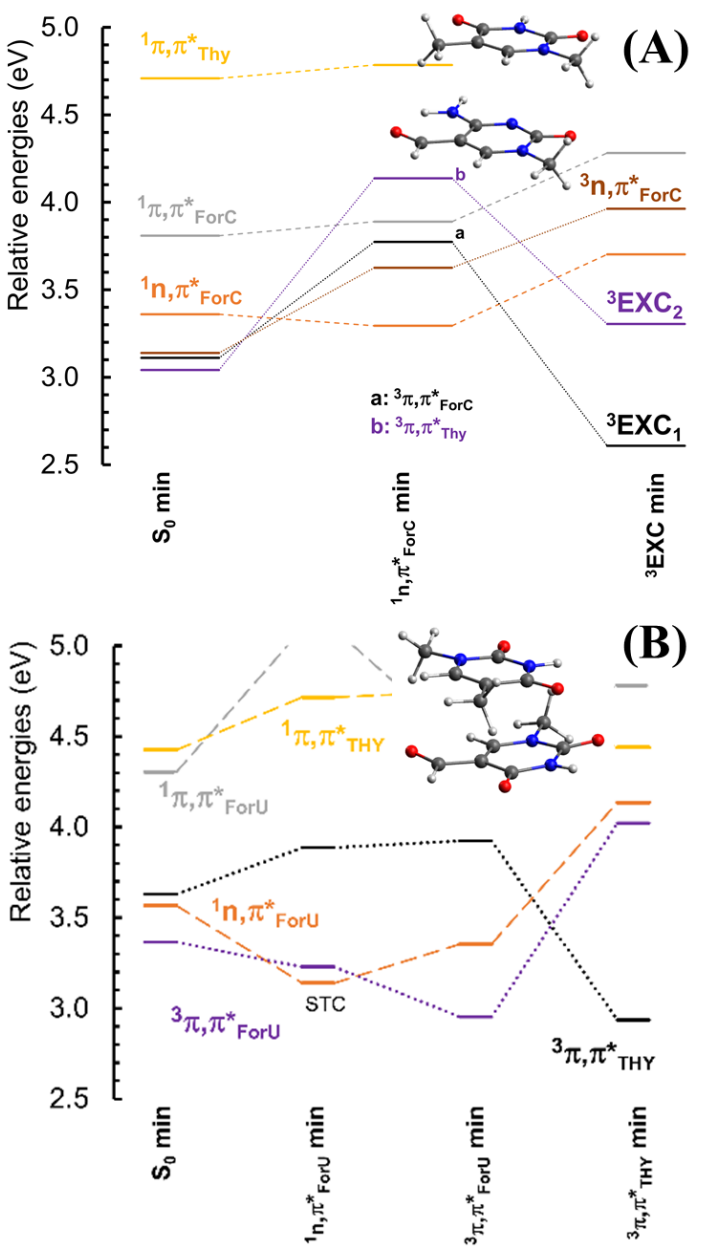

Figure 5. (A) CASPT2 energy profiles for the most relevant excited states of the ForC-Thy system. (B) CASPT2 energy profiles for the most relevant excited states of the ForU-Thy system, taken from Ref. ${ }^{35}$. STC = singlet-triplet crossing. The horizontal axis defines the equilibrium geometry of the most important states, obtained via geometry optimization, the dashed lines are used to connect the states having the same diabatic nature as a guide for the eyes.

electronic states, with a small but non-negligible driving force favoring the transfer of spin density to Thy, in a process requiring to bypass only a relatively small energy barrier.

The presence of the low energy ${ }^{3} \mathrm{EXC}_{2}$ in ForC can contribute to further explain the different observed yields. Indeed, the possibility of the formation of a delocalized state involving the sensitizer and two thymine bases attached to a rigid scaffold has been previously invoked in the case of benzophenone, another photosensitizer. ${ }^{41}$ Different mechanisms could be at play here (see Scheme 2) for ForC, in which (i) the exciplex state is too stable to be dissociated, and act as the energy donor for TTET to Thy, however the decreased energy of ${ }^{3} \mathrm{EXC}_{2}(2.61 \mathrm{eV})$ locates this state below of Thy triplet excited state (at $2.87 \mathrm{eV})^{21}$, which makes TTET process unlikely; or (ii) the exciplex ${ }^{3} \mathrm{EXC}_{2}$ can eventually further delocalize involving a second Thy unit forming a triplet triplex 


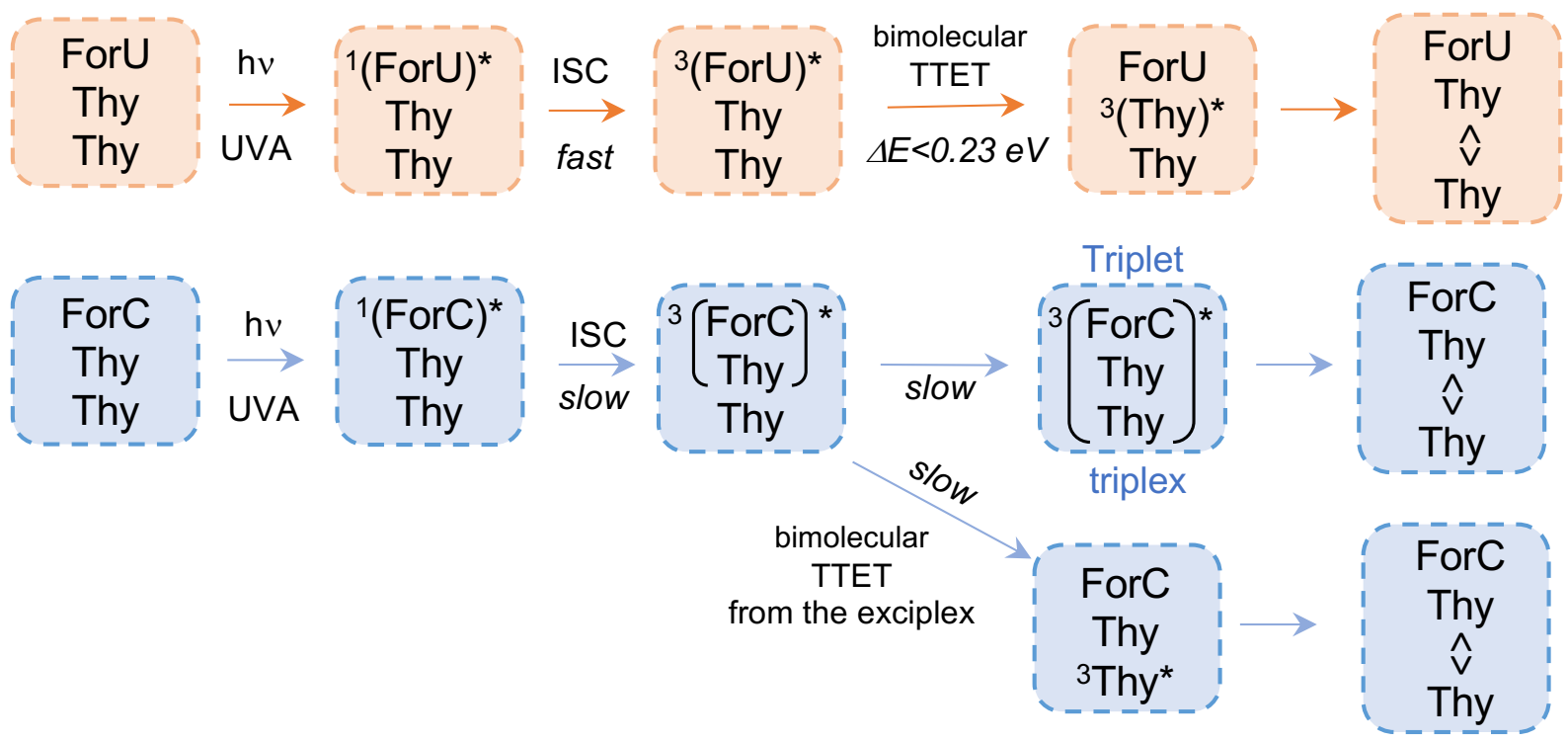

Scheme 2. Schematic representation of the proposed CPD production from excitation of ForU (top) and ForC (bottom), according to our experimental and computational data. ISC = intersystem crossing, TTET = triplet-triplet energy transfer.

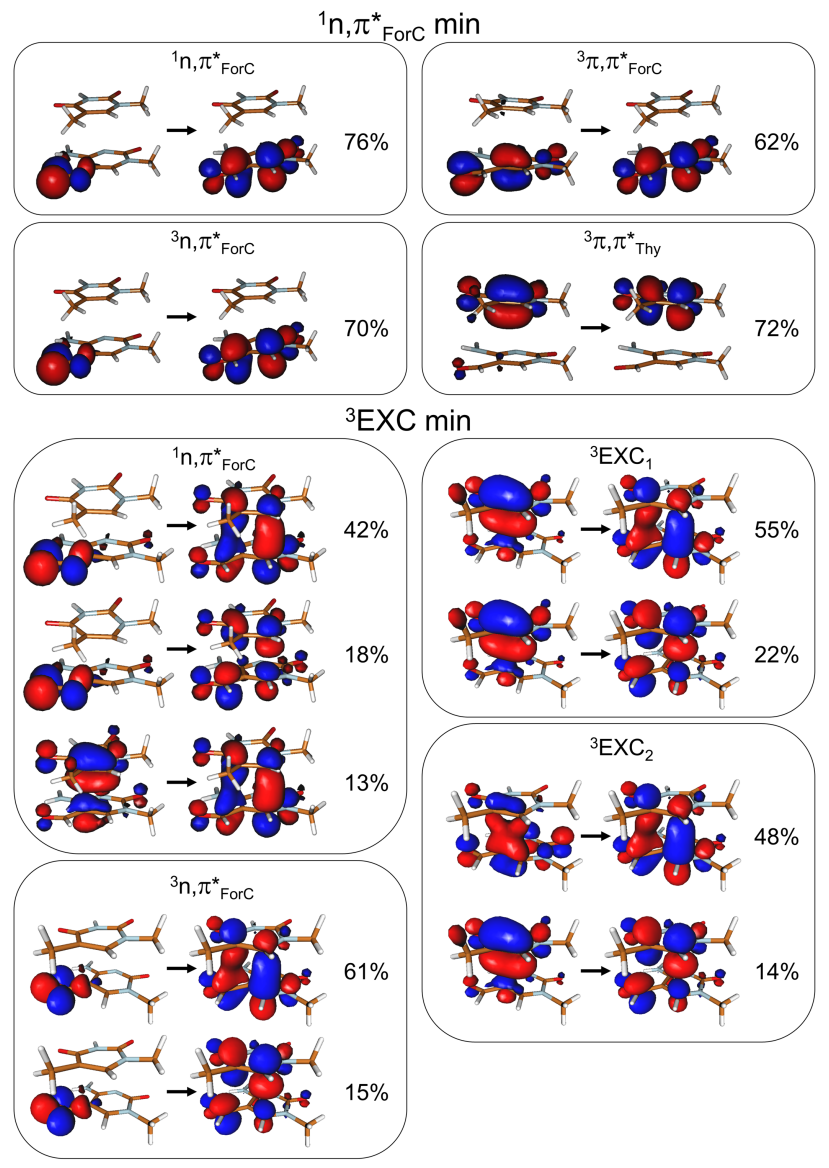

Figure 6. CASSCF orbitals corresponding to the highest weight electronic transitions that characterize the excited states of the ForC-Thy system. The weights of the configurations are also shown. Other transitions with much lower weights adding up to the total $100 \%$ are not reported. that evolves forming Thy<>Thy, nonetheless, such a trimolecular process can clearly be seen as slower and less efficient than the most direct route operative in the case of ForU involving localized states (Scheme 2).

Globally, the formation of the exciplex state as well as the trapping of the system in the ${ }^{1} n, \pi^{*}$ Forc minimum, decrease the overall efficiency of the photoprocess in the ForC system.

\section{CONCLUSIONS}

The present work characterizes the formation of dangerous CPD lesions through triplet-triplet energy transfer from ubiquitous oxidized nucleobases and epigenetic intermediates. The differences of the intrinsic photosensitization capabilities of ForU and ForC are revealed in detail by timeresolved and steady-state absorption spectroscopy, NMR, HPLC, and molecular modeling. It has been observed that the triplet-triplet energy transfer rate from ForU to thymine-thymine is 3 times faster than that from $\operatorname{ForC}\left(\mathrm{k}_{\mathrm{q}} \sim 1.3\right.$ $\mathrm{x} 10^{9} \mathrm{M}^{-1} \mathrm{~s}^{-1}$ for ${ }^{3} \mathrm{ForU} \mathrm{U}^{*}$ and $4.6 \times 10^{8} \mathrm{M}^{-1} \mathrm{~s}^{-1}$ for ${ }^{3} \mathrm{ForC} \mathrm{C}^{*}$ ), leading to a complete depletion of the thymine-thymine reactant after 1 hour of irradiation in the presence of ForU. Under the same conditions, the consumption of the reactant is only about 50\% when ForC acts as photosensitizer. Molecular modelling provides further evidence supporting a slower intersystem crossing and triplet-triplet energy transfer in ForC, since the relaxation of the singlet excited state leads to a region where the triplet states are less accessible. Exciplex states of ${ }^{3} \pi, \pi^{*}\left({ }^{3} \mathrm{EXC}\right)$ nature dominate the triplet state manifold in ForC-Thy and are identified as mediators of the triplet-triplet energy transfer in this system.

These results represent a step forward in the understanding of the intimate coupling between oxidative and light-induced DNA damage, responsible of the public health problem of unprotected sunlight exposure and development of 
malignant skin diseases. Moreover, we also believe these results could be useful in the context of tumor detection and light-assisted therapies, since the different capability of ForC and ForU in inducing measurable CPD lesions could be used to determine or estimate the ratio of both species in DNA samples, especially relevant taking into account that their biological role is different and thus are involved in different processes.

\section{ASSOCIATED CONTENT}

Materials and methods, full computational details, synthetic details and characterization of Thy-Thy and Thy<> Thy, phosphorescence emission, decays of ForC in the presence of ThyThy, NMR analysis of [2+2] photocycloaddition from irradiation of Thy-Thy, HPLC analysis of irradiation of Thy-Thy. This material is available free of charge via the Internet at http://pubs.acs.org.

\section{AUTHOR INFORMATION}

\section{Corresponding Author}

*A.M.: antonio.monari@univ-lorraine.fr, M.A.M.: $\underline{\text { mmi- }}$ randa@qim.upv.es, V.L.-V.: lvirgini@itq.upv.es

\section{ACKNOWLEDGMENT}

Support from the Université de Lorraine, CNRS, regional (Prometeo/2017/075) and Spanish Government (PGC2018096684-B-I00, CTQ2017-87054-C2-2-P) is kindly acknowledged. A. F.-M. is grateful to Generalitat Valenciana and the European Social Fund for a postdoctoral contract (APOSTD/2019/149), M. L.-R. acknowledges the Universitat Politècnica de València for the FPI grant. All calculations have been performed on the local LPCT computer center and on the Explor regional center in the framework of the project "Dancing under the light".

\section{REFERENCES}

(1) Crespo-Hernandez, C. E.; Cohen, B.; Hare, P. M.; Kohler, B. Ultrafast Excited-State Dynamics in Nucleic Acids. Chem. Rev 2004, $104 \quad$ (4), 1977-2019. https://doi.org/10.1021/cr0206770.

(2) Improta, R.; Santoro, F.; Blancafort, L. Quantum Mechanical Studies on the Photophysics and the Photochemistry of Nucleic Acids and Nucleobases. Chem. Rev. 2016, pp 35403593. https://doi.org/10.1021/acs.chemrev.5b00444.

(3) Sage, E.; Girard, P.-M.; Francesconi, S. Unravelling UVAInduced Mutagenesis. Photochem. Photobiol. Sci. 2012, 11 (1) 74-80. https://doi.org/10.1039/C1PP05219E.

(4) Francés-Monerris, A.; Gattuso, H.; Roca-Sanjuán, D.; Tuñón, I.; Marazzi, M.; Dumont, E.; Monari, A. Dynamics of the ExcitedState Hydrogen Transfer in a (DG).(DC) Homopolymer: Intrinsic Photostability of DNA. Chem. Sci. 2018, 9 (41), 79027911. https://doi.org/10.1039/c8sc03252a.

(5) Zhang, Y.; De La Harpe, K.; Beckstead, A. A.; Improta, R. Kohler, B. UV-Induced Proton Transfer between DNA Strands. J. Am. Chem. Soc. 2015, 137 (22), 7059-7062. https://doi.org/10.1021/jacs.5b03914.

(6) Zhang, Y.; Li, X.-B.; Fleming, A. M.; Dood, J.; Beckstead, A. A.; Orendt, A. M.; Burrows, C. J.; Kohler, B. UV-Induced ProtonCoupled Electron Transfer in Cyclic DNA Miniduplexes. J. Am. Chem. Soc. 2016, 138 (23), 7395-7401. https://doi.org/10.1021/jacs.6b03216.

(7) Bucher, D. B.; Schlueter, A.; Carell, T.; Zinth, W. Watson-Crick Base Pairing Controls Excited - State Decay in Natural DNA. Angew. Chem. Int. Ed. 2014, 53 (42), 11366-11369. https://doi.org/10.1002/anie.201406286.
Rottger, K.; Marroux, H. J. B.; Grubb, M. P.; Coulter, P. M.; Bohnke, H.; Henderson, A. S.; Galan, M. C.; Temps, F.; OrrEwing, A. J.; Roberts, G. M. Ultraviolet Absorption Induces Hydrogen-Atom Transfer in G Center Dot C Watson-Crick DNA Base Pairs in Solution. Angew. Chemie-International Ed. 2015, 54 (49), 14719-14722. https://doi.org/10.1002/anie.201506940.

(9) Nogueira, J. J.; Plasser, F.; Gonzalez, L. Electronic Delocalization Charge Transfer And Hypochromism in the UV Absorption Spectrum of Polyadenine Unravelled by Multiscale Computations and Quantitative Wavefunction Analysis. Chem. Sci. 2017, 8 (8), 5682-5691. https://doi.org/10.1039/C7SC01600J.

(10) Barbatti, M.; Aquino, A. J. A.; Szymczak, J. J.; Nachtigallová, D. Hobza, P.; Lischka, H. Relaxation Mechanisms of UVPhotoexcited DNA and RNA Nucleobases. Proc. Natl. Acad. Sc. U.S.A. 2010, $107 \quad$ (50), 21453-21458. https://doi.org/10.1073/pnas.1014982107.

(11) Reiter, S.; Keefer, D.; De Vivie-Riedle, R. RNA Environment Is Responsible for Decreased Photostability of Uracil. J. Am. $\begin{array}{llll}\text { Chem. Soc. 2018, } 140 & \text { (28), 8714-8720 }\end{array}$ https://doi.org/10.1021/jacs.8b02962. Photosensitised Pyrimidine Dimerisation in DNA. Chem. Sci. 2011, 2, 1219-1232. https://doi.org/10.1039/c1sc00088h. Mouret, S.; Baudouin, C.; Charveron, M.; Favier, A.; Cadet, J.; Douki, T. Cyclobutane Pyrimidine Dimers Are Predominant DNA Lesions in Whole Human Skin Exposed to UVA Radiation. Proc. Natl. Acad. Sci. U. S. A. 2006, 103 (37), 1376513770. https://doi.org/10.1073/pnas.0604213103.

Ikehata, H.; Mori, T.; Kamei, Y.; Douki, T.; Cadet, J.; Yamamoto, M. Wavelength- and Tissue-Dependent Variations in the Mutagenicity of Cyclobutane Pyrimidine Dimers in Mouse Skin. Photochem. Photobiol. 2020, 96 (1), 94-104. https://doi.org/10.1111/php.13159.

(15) Pfeifer, G. P.; Besaratinia, A. UV Wavelength-Dependent DNA Damage and Human Non-Melanoma and Melanoma Skin Cancer. Photochem. Photobiol. Sci. 2012, 11 (1), 90-97. https://doi.org/10.1039/c1pp05144j.

(16) Noonan, F. P.; Zaidi, M. R.; Wolnicka-Glubisz, A.; Anver, M. R.; Bahn, J.; Wielgus, A.; Cadet, J.; Douki, T.; Mouret, S.; Tucker, M. A.; Popratiloff, A.; Merlino, G.; De Fabo, E. C. Melanoma Induction by Ultraviolet A but Not Ultraviolet B Radiation Requires Melanin Pigment. Nat. Commun. 2012, 3, 884. https://doi.org/10.1038/ncomms1893.

(17) Sinha, R. P.; Häder, D.-P. UV-Induced DNA Damage and Repair: A Review. Photochem. Photobiol. Sci. 2002, 1 (4), 225-236. https://doi.org/10.1039/b201230h.

(18) Epe, B. DNA Damage Spectra Induced by Photosensitization Photochem. Photobiol. Sci. 2012, 11 (1), 98-106. https://doi.org/10.1039/C1PP05190C.

(19) Francés-Monerris, A.; Tuñón, I.; Monari, A. Hypoxia-Selective Dissociation Mechanism of a Nitroimidazole Nucleoside in a DNA Environment. J. Phys. Chem. Lett. 2019, 10 (21), 67506754. https://doi.org/10.1021/acs.jpclett.9b02760.

(20) Roca-Sanjuán, D.; Olaso-González, G.; González-Ramírez, I.; Serrano-Andrés, L.; Merchán, M. Molecular Basis of DNA Photodimerization: Intrinsic Production of Cyclobutane Cytosine Dimers. J. Am. Chem. Soc. 2008, 130 (32), 1076810779. https://doi.org/10.1021/ja803068n.

(21) Climent, T.; González-Ramírez, I.; González-Luque, R.; Merchán, M.; Serrano-Andrés, L. Cyclobutane Pyrimidine Photodimerization of DNA/RNA Nucleobases in the Triplet State. J. Phys. Chem. Lett. 2010, 1 (14), 2072-2076. https://doi.org/10.1021/jz100601p.

(22) Dumont, E.; Monari, A. Understanding DNA under Oxidative Stress and Sensitization: The Role of Molecular Modeling. Front. Chem. 2015, 3, 43 . https://doi.org/10.3389/fchem.2015.00043.

(23) Lhiaubet-Vallet, V.; Sarabia, Z.; Hernández, D.; Castell, J. V.; Miranda, M. A. In Vitro Studies on DNA-Photosensitization by Different Drug Stereoisomers. Toxicol. Vitr. 2003, 17 (5-6) https://doi.org/10.1016/S0887-2333(03)00108-5. 
J. Analysis of Fluoroquinolone-Mediated Photosensitization of 2'-Deoxyguanosine, Calf Thymus and Cellular DNA Determination of Type-I, Type-II and Triplet-Triplet Energy Transfer Mechanism Contribution 1 . Photochem. Photobiol. 2001, 73 (3), 230-237. https://doi.org/10.1562/00318655(2001)0730230AOFMP02.0.CO2.

(25) Lhiaubet-Vallet, V.; Bosca, F.; Miranda, M. A. Photosensitized DNA Damage: The Case of Fluoroquinolones. Photochem. Photobiol. 2009, 85 (4), 861-868. https://doi.org/10.1111/j.1751-1097.2009.00548.x.

(26) Cuquerella, M. C.; Lhiaubet-Vallet, V.; Cadet, J.; Miranda, M. A. Benzophenone Photosensitized DNA Damage. Acc. Chem. Res. 2012, $45 \quad$ (9), 1558-1570. https://doi.org/10.1021/ar300054e.

(27) Marazzi, M.; Mai, S.; Roca-Sanjuán, D.; Delcey, M. G.; Lindh, R.; González, L.; Monari, A. Benzophenone Ultrafast Triplet Population: Revisiting the Kinetic Model by Surface-Hopping Dynamics. J. Phys. Chem. Lett. 2016, 7 (4), 622-626. https://doi.org/10.1021/acs.jpclett.5b02792.

(28) Dumont, E.; Wibowo, M.; Roca-Sanjuán, D.; Garavelli, M.; Assfeld, X.; Monari, A. Resolving the Benzophenone DNAPhotosensitization Mechanism at QM/MM Level. J. Phys. Chem. Lett. 2015, 6 (4), 576-580. https://doi.org/10.1021/jz502562d.

(29) Vendrell-Criado, V.; Rodriguez-Muniz, G. M.; Cuquerella, M. C.; Lhiaubet-Vallet, V.; Miranda, M. A. Photosensitization of DNA by 5-Methyl-2-Pyrimidone Deoxyribonucleoside: (6-4) Photoproduct as a Possible Trojan Horse. Angew. Chem. Int. Ed. 2013, 52 (25), 6476-6479. https://doi.org/10.1002/anie.201302176.

(30) Bignon, E.; Gattuso, H.; Morell, C.; Dumont, E.; Monari, A. DNA Photosensitization by an "Insider": Photophysics and Triplet Energy Transfer of 5-Methyl-2-Pyrimidone Deoxyribonucleoside. Chem. Eur. J. 2015, 21 (32), 1150911516. https://doi.org/10.1002/chem.201501212.

(31) Rogstad, D. K.; Heo, J.; Vaidehi, N.; Goddard, W. A.; Burdzy, A.; Sowers, L. C. 5-Formyluracil-Induced Perturbations of DNA Function. Biochemistry 2004, 43 (19), 5688-5697. https://doi.org/10.1021/bi030247j.

(32) Bachman, M.; Uribe-Lewis, S.; Yang, X.; Burgess, H. E.; Iurlaro, M.; Reik, W.; Murrell, A.; Balasubramanian, S. 5Formylcytosine Can Be a Stable DNA Modification in Mammals. Nat. Chem. Biol. 2015, 11 (8), 555-557. https://doi.org/10.1038/nchembio.1848.

(33) Wang, Y.; Zhang, X.; Zou, G.; Peng, S.; Liu, C.; Zhou, X. Detection and Application of 5-Formylcytosine and 5-Formyluracil in DNA. Acc. Chem. Res. 2019, 52 (4), 1016-1024. https://doi.org/10.1021/acs.accounts.8b00543.

(34) Aparici-Espert, I.; Garcia-Lainez, G.; Andreu, I.; Miranda, M. A.; Lhiaubet-Vallet, V. Oxidatively Generated Lesions as Internal Photosensitizers for Pyrimidine Dimerization in DNA. ACS Chem. Biol. 2018, 13, 542-547. https://doi.org/10.1021/acschembio.7b01097.

(35) Francés-Monerris, A.; Hognon, C.; Miranda, M. A.; LhiaubetVallet, V.; Monari, A. Triplet Photosensitization Mechanism of Thymine by an Oxidized Nucleobase: From a Dimeric Model to DNA Environment. Phys. Chem. Chem. Phys. 2018, 20 (40), 25666-25675. https://doi.org/10.1039/C8CP04866E.

(36) Wang, X.; Yu, Y.; Zhou, Z.; Liu, Y.; Yang, Y.; Xu, J.; Chen, J. Ultrafast Intersystem Crossing in Epigenetic DNA Nucleoside 2'-Deoxy-5-Formylcytidine. J. Phys. Chem. B 2019, 123 (27), 5782-5790.

(37) Francés-Monerris, A.; Lineros-Rosa, M.; Miranda, M. A.; Lhiaubet-Vallet, V.; Monari, A. Photoinduced Intersystem Crossing in DNA Oxidative Lesions and Epigenetic Intermediates. Chem. Commun. 2020, 56 (32), 4404-4407. https://doi.org/10.1039/d0cc01132k.

(38) Lhiaubet-Vallet, V.; Miranda, M. A. CRC Handbook of Organic Photochemistry and Photobiology. In CRC Handbook of Organic Photochemistry and Photobiology; Ghetti, F., Griesbeck, A. G., Oelgemöller, M., Eds.; CRC Press, 2012; pp 1541-1555.

(39) Alzueta, O. R.; Cuquerella, M. C.; Miranda, M. A. Triplet Energy Transfer versus Excited State Cyclization as the Controlling Step in Photosensitized Bipyrimidine Dimerization. 2019. https://doi.org/10.1021/acs.joc.9b01423.

(40) Lhiaubet-Vallet, V.; Cuquerella, M. C.; Castell, J. V.; Bosca, F.; Miranda, M. A. Triplet Excited Fluoroquinolones as Mediators for Thymine Cyclobutane Dimer Formation in DNA. J. Phys. Chem. B 2007, 111 (25). https://doi.org/10.1021/jp070167f Miro, P.; Lhiaubet-Vallet, V.; Marin, M. L.; Miranda, M. A Photosensitized Thymine Dimerization via Delocalized Triplet Excited States. Chem. Eur. J. 2015, 21 (47), 1705117056. https://doi.org/10.1002/chem.201502719. 
Authors are required to submit a graphic entry for the Table of Contents (TOC) that, in conjunction with the manuscript title, should give the reader a representative idea of one of the following: A key structure, reaction, equation, concept, or theorem, etc., that is discussed in the manuscript. Consult the journal's Instructions for Authors for TOC graphic specifications.

Insert Table of Contents artwork here 